\title{
Accumulation and modeling of particles in drinking water pipe fittings
}

\author{
K. Neilands, M. Bernats, and J. Rubulis \\ Riga Technical University, Department of Water Engineering and Technology, Latvia \\ Correspondence to: K. Neilands (kaspars.neilands@rtu.lv) \\ Received: 16 February 2012 - Published in Drink. Water Eng. Sci. Discuss.: 11 April 2012 \\ Revised: 6 August 2012 - Accepted: 7 August 2012 - Published: 3 September 2012
}

\begin{abstract}
The effect of pipe fittings (mainly T-pieces) on particle accumulation in drinking water distribution networks were shown in this work. The online measurements of flow and turbidity for cast iron, polyethylene and polyvinyl chloride pipe sections were linked with analysis of pipe geometry. Up to $0.29 \mathrm{~kg}$ of the total amount mobilized in T-pieces ranging from DN 100/100-DN 250/250. The accumulated amount of particles in fittings was defined as $J$ and introduced into the existing turbidity model PODDS (prediction of discoloration in distribution systems) proposed by Boxall et al. (2001) which describes the erosion of particles leading to discoloration events in drinking water network viz sections of straight pipes. However, this work does not interpret mobilization of particles in pipe fittings which have been considered in this article. T-pieces were the object of this study and depending of the diameter or daily flow velocity, the coefficient $J$ varied from 1.16 to 8.02. The study showed that pipe fittings act as catchment areas for particle accumulation in drinking water networks.
\end{abstract}

\section{Introduction}

The accumulation of particles in drinking water distribution networks is commonly observed. A considerable number of studies have been done on explaining fundamentals of processes (van Thienen et al., 2011a; Boxall et al., 2003), a mathematical description (Ryan et al., 2008; Boxall et al., 2001) and management advices (Vreeburg et al., 2004; Matsui et al., 2007; Saldarriaga et al., 2010; Husband et al., 2010) regarding water discoloration in a water supply network has been achieved.

A detailed work (Ryan et al., 2008) was carried out in order to explain the mechanisms responsible for particle accumulation in drinking water networks. However, in the proposed particle sediment model (PSM) only the mechanism of gravitational force of particles where the threshold value for deposition is set as $0.07 \mathrm{~m} \mathrm{~s}^{-1}$ and for resuspension $0.2 \mathrm{~m} \mathrm{~s}^{-1}$ (in $100 \mathrm{~mm}$ pipe) was included. As a second mechanism, the deposition of particles on the pipe walls was described (Ryan et al., 2008), which could affect the deposition by the van der Walls forces which are known to be weak; in this case the small distance between the particles and pipe surface is important. Fick's law was used to describe diffusion of particles from bulk flow to pipe wall. Although this mechanism was not included in the model, from the experimental results it was apparent that the particles (specific gravity 1.64) precipitated better on polyvinyl chloride (PVC) than on cement lined metal pipe. The concentration of particles on PVC pipe wall is more sensitive to flow velocity, which do not include region of gravitational settling $\left(0.07-0.3 \mathrm{~m} \mathrm{~s}^{-1}\right)$ (Ryan et al., 2008). The amount of particles deposited on the wall of the PVC pipes was shown to be larger than the amount deposited on the wall of the cement lined metal pipes, for the same working conditions (velocity, initial concentration). Although the lab-scale experiments were done in pipe loop (length $=41 \mathrm{~m}$ ) consisting of four $90^{\circ}$ bends, there was no information on any influence of loop geometry on the accumulation results (Ryan et al., 2008).

The application of the stochastic water demand model SIMDEUM (SIMulation of Demand, an End-Use Model) together with turbidity measurements from a uni-directional flushing (UDF) program showed that maximum daily velocities in range $0.05-0.1 \mathrm{~m} \mathrm{~s}^{-1}$ give the highest concentrations of particles in a network (Blokker et al., 2010). While it has 
been concluded that maximum daily velocity is not the only parameter responsible for particle accumulation, the role of radial transport processes can be explained by, for example, turbophoresis and turbulent diffusion, which was based on the laboratory-scale experiments (van Thienen et al., 2011b) in which the deposition of particles on the entire wall circumference at velocity $0.14 \mathrm{~m} \mathrm{~s}^{-1}$ with particles of specific gravity 1.2 was demonstrated. To describe these processes the virtual mass effect which refers to inertia of particles in water together with the Magnus and the Saffman forces, which describe the rotation of a particle in water, was discussed regarding the particle accumulation process in pipe sections. The authors have not taken a closer look at the geometry; remarked that the internal irregularities in the distribution pipe system may be catchment areas for particles. It should be noted that particles from wash-out during first flushing turnover mobilized along the entire pipe length can be called locally settled sediments (LSS) (Blokker et al., 2010).

Boxall et al. (2001), Boxall and Saul (2005) proposed the PODDS (prediction of discolouration in distribution systems) model by involving the term "cohesive transport model" where discoloration material is assumed to be homogenously distributed around the pipes circumference in cohesive layers of particulate matter. The layer strength is a function of the maximum daily shear stress. Material erosion and regeneration processes can be modelled through calibration. Proposed empirical equations for the description of erosion have been verified in lab-scale polyethylene (PE) pipe loop (length $=600 \mathrm{~m}$ ) with an elliptical form (Husband et al., 2008), and in both plastic and rough corroded metal pipes with different diameters in field conditions (Husband and Boxall, 2010). The shear stress and subsequent erosion of particles increased with the flow. It is considered that the accumulation of particles occurs in layers around the complete pipe circumference which is conditioned via daily hydraulic flow. The accumulation mechanisms via corrosion (direct) and the background concentration of material from upstream or the treatment plant (indirect) have been discussed (Husband and Boxall, 2010). The origin of the particles in distribution network is has been widely described (Gauthier et al., 1999; Verberk et al., 2006; Husband et al., 2008; Vreeburg, 2007; Vreeburg et al., 2008); although , this is not the subject of the current paper. There is some criticism expressed towards the PODDS model, e.g. that (1) the formulations of the equations which predict the accumulation rate of particles should be subordinated to the fundamental laws (van Thienen et al., 2011a) and (2) the parameter that the rate of change of the velocity or, subsequently, unsteady shear stress is applied during the early stage of hydraulic transient event (e.g. fast valve opening/closing) plays a significant role for the first seconds of erosion of the particles (Aisopou et al., 2010). It should be mentioned that PODDS supports the cohesive layer assumptions in the approach considering the entire pipe length irrespective of the topographical features, while afterwards, some irregularities in washed out particle concentration obtained during the trunk main flushing within section consisting of four $90^{\circ}$ bends and depressions in the pipe topography have been noted (Husband et al., 2010).

The particle accumulation and the subsequent erosion within the drinking water pipe networks has been broadly described within the past few years. However, there is a lack of information regarding the particle concentration in locations where water flow changes due to geometry of conventional fittings (T-pieces, valves, bends etc.). In a recent study, we reported the analysis of detailed pipe geometry (topology) and online turbidity curves obtained from particle erosion during UDF via one and two hydrants simultaneously, and demonstrated that these spikes corresponded to location of network fittings (Rubulis and Neilands, 2010).

In this work the influence of pipe geometry in accumulation process of particles in drinking water distribution networks has been investigated. The major objectives of this study were to (1) analyze UDF data from field events with different diameter T-pieces and other fittings; (2) link UDF data (shear stress, turbidity and mass) with equations of PODDS by using MS Excel to model the erosion of particles; (3) explain the field measurements using an approach from tracer studies regarding the particle transport in other industrial applications.

\section{Materials and methods}

\subsection{Description of study site}

The UDF operation was performed in a town with population of about 9000 inhabitants. The overall network consists of $13 \mathrm{~km}$ of cast iron (CI) material, of $4 \mathrm{~km}$ of PVC and $3 \mathrm{~km}$ of polyethylene (PE) pipes (Fig. 1).

The CI pipes were mainly constructed between the years 1970-1990, while PVC and PE pipes were built during the years 2000-2011. For the supply of drinking water networks the treatment with aeration followed by rapid sand filtration of $700 \mathrm{~m}^{3} \mathrm{day}^{-1}$ has been applied. The water quality outlet of the plant from the grab sample program summarized in Table 1 is characteristic of corrosive water with a high salt concentration. Neilands et al. (2009) proved that the application of the online monitoring system reveals the problems with the particle load in the network due to a deficient operation of rapid sand filters during the flushing process in the morning hours (Fig. 2).

The network had not been flushed before. The accumulation of sediments in the network was assessed with the resuspension potential method (RPM) proposed by Vreeburg et al. (2004) which revealed considerable risk of discoloration (Table 2).

\subsection{Experimental design of field measurements}

In general, the online turbidity data from UDF were linked with a careful analysis of technical drawings of each flushed 


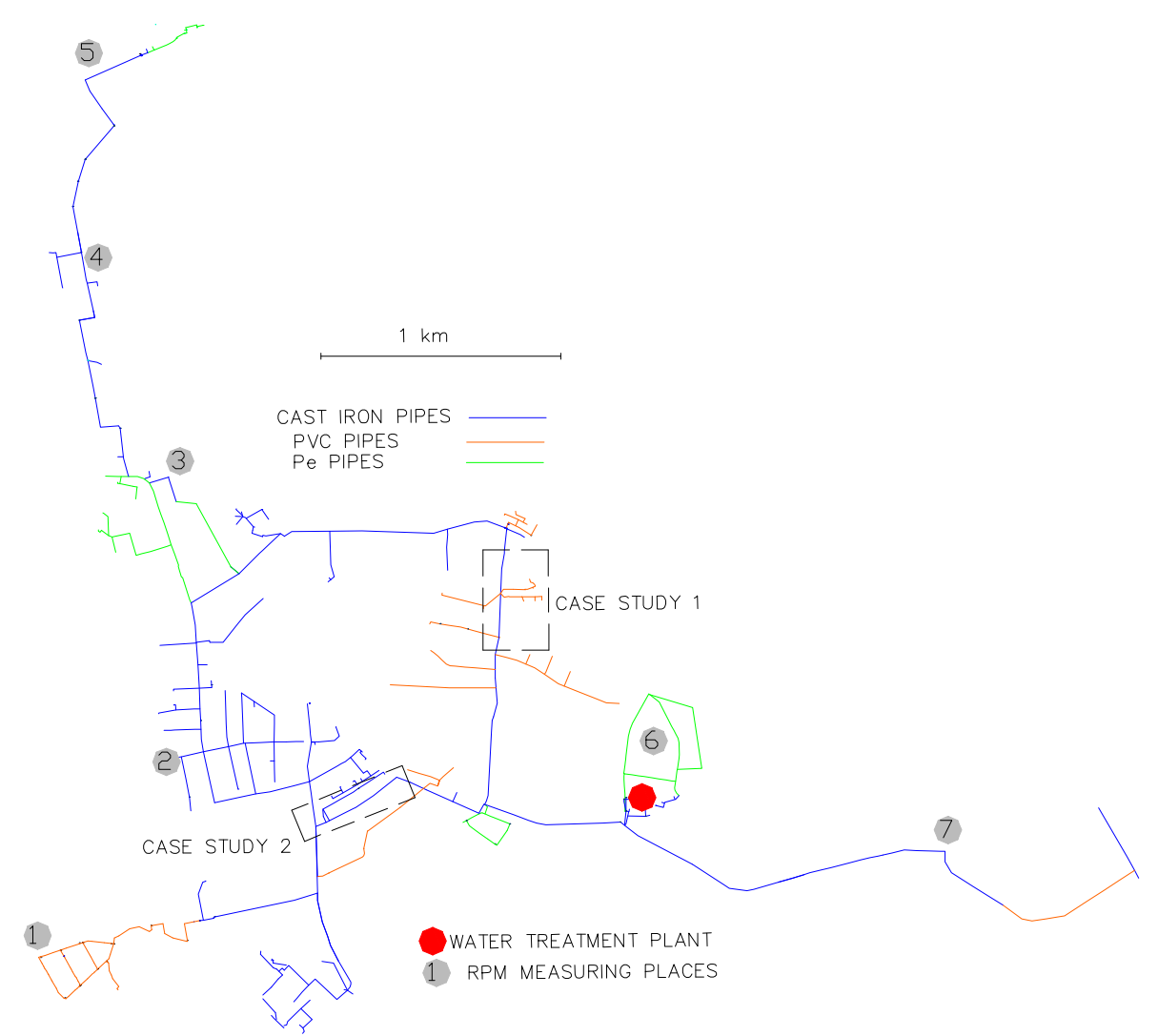

Figure 1. The diagram of the drinking water network indicating numerated circles for locations of resuspension potential method. Flushing sections are indicated with a dashed line. CI pipes are shown in blue, the PVC pipes are shown in orange and the PE pipes are shown in green.

Table 1. The water quality in water works (treatment plants). The average results with standard deviations and coefficient of variation (in brackets) are shown.

\begin{tabular}{ll}
\hline Parameter, units & Average results \\
\hline Conductivity, $\mu \mathrm{S} \mathrm{cm}^{-1}$ & $739 \pm 16(20)$ \\
$\mathrm{pH}$ & $6.97 \pm 0.38(18)$ \\
Iron (Fe tot), $\mathrm{mg} \mathrm{l}^{-1}$ & $0.05 \pm 0.02(4)$ \\
Iron (FeII), $\mathrm{mg} \mathrm{l}^{-1}$ & $0.01 \pm 0.01(4)$ \\
Turbidity, NTU & $0.344 \pm 0.32(94)$ \\
Chlorine (tot) $\mathrm{mg} \mathrm{l}^{-1}$ & $0.038 \pm 0.033(17)$ \\
Chlorine (free), $\mathrm{mg} \mathrm{l}^{-1}$ & $0.012 \pm 0.021(8)$ \\
Langelier Index & -1.23 \\
Red-ox, mV & $204 \pm 10(17)$ \\
\hline
\end{tabular}

pipe section while paying particular attention to the location of the fitting. In total, 67 isolated pipe sections were flushed. The turbidity data during the flushing process were recorded for at least every minute (in several cases even $5 \mathrm{~s}$ ) using calibrated online photometer ULTRATURB plus ${ }^{\mathrm{TM}}$ equipped with a controller sc $100^{\mathrm{TM}}$ (Dr. Bruno Lange GmbH\&Co, Düsselfdorf, Germany) installed into a flushing vehicle equipped with a manometer and the Siemens Sitrans FM MAG 8000 online flow meter (Siemens Energy \& Automation Inc., Germany). The standard deviation for measurements was $\pm 30 \mathrm{~s}$, depending on how fast the necessary flow rate was reached during the opening of the fire hydrant. Mechanical cleaning of the measuring chamber in addition to the automatic cleaning of the measuring chamber of ULTRATURB plus ${ }^{\mathrm{TM}}$ was routinely performed.

The clear PVC pipe segment (inner $\emptyset 75 \mathrm{~mm}$ ) in the flushing vehicle was connected with a $1 \mathrm{~m}$ long fire hose (inner $\varnothing 75 \mathrm{~mm}$ ), which was connected directly to the standpipe of the hydrant. The construction of the flushing vehicle was done assuming that the water flow from the standpipe would have the shortest possible path till the online turbidity meter. The online turbidity measurements during UDF were controlled with simultaneous turbidity measurements in grab samples performed with a portable manual turbidity meter Hach 2100P (Dr. Bruno Lange GmbH\&Co, Düsselfdorf, Germany). This was done in a synchronized manner with all the measuring equipment at the start of the flushing. 


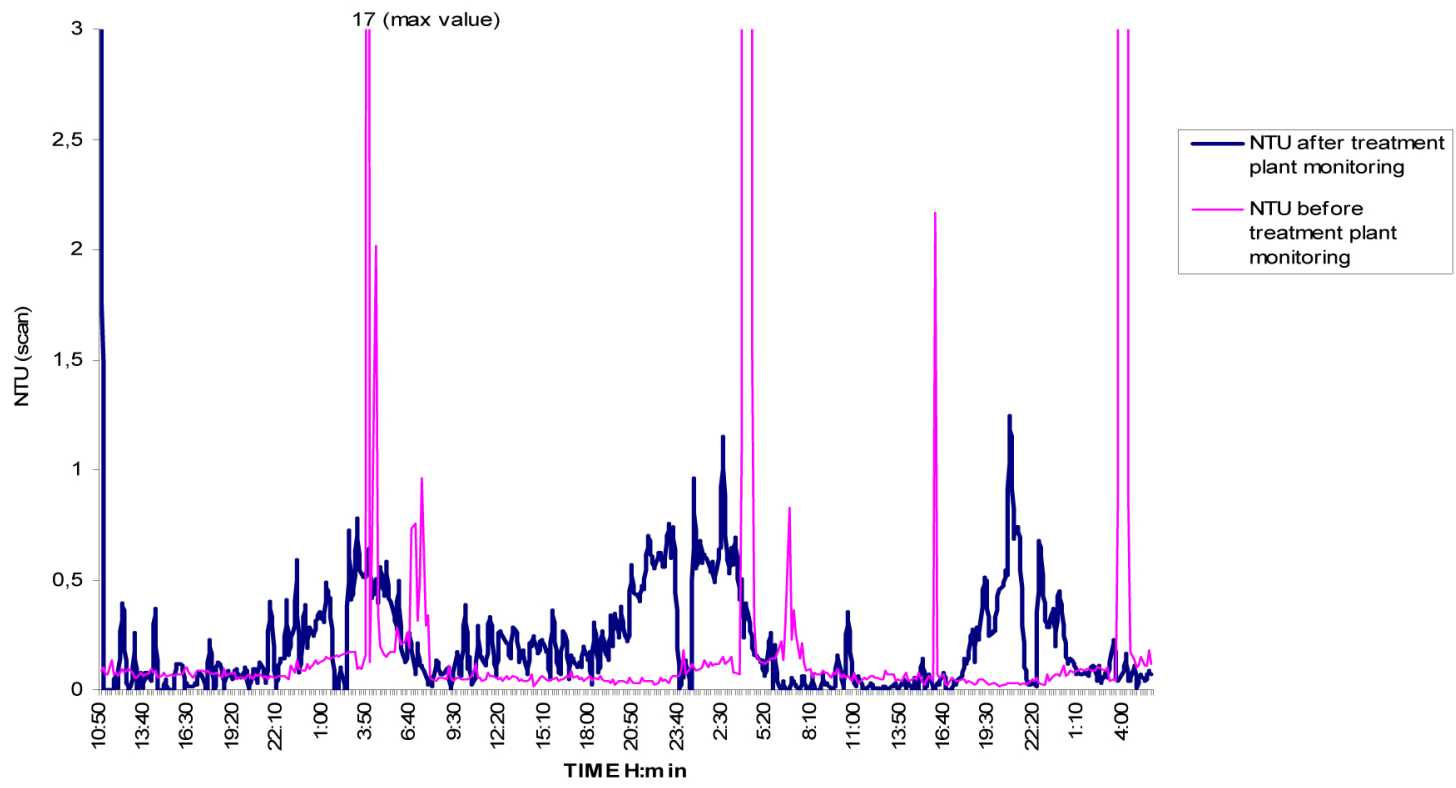

Figure 2. The online measurements of turbidity in the outlet of the WTP of the distribution network before and after the repair of the rapid sand filters. Values on the top of figure show the peaks of turbidity. Modified from Neilands et al. (2009).

Table 2. The results of RPM ranking measured with Ultraturb ${ }^{\mathrm{TM}}$ equipment in the field study place (Fig. 1).

\begin{tabular}{ccccrr}
\hline Nr. & Diameter & Material & $\begin{array}{c}\text { Section } \\
\text { length } \\
(\mathrm{m})\end{array}$ & RPM & $\begin{array}{r}\text { Average } \\
\text { turbidity } \\
(\mathrm{NTU})\end{array}$ \\
\hline 1 & 100 & PVC & 326 & 14 & 114.6 \\
2 & 150 & $\mathrm{CI}$ & 326 & 14 & 36.0 \\
3 & 150 & $\mathrm{CI}$ & 397 & 7 & 6.9 \\
4 & 150 & $\mathrm{CI}$ & 423 & 5 & 3.8 \\
5 & 150 & $\mathrm{CI}$ & 401 & 10 & 10.7 \\
6 & 200 & $\mathrm{PE}$ & 324 & 15 & 105.8 \\
7 & $100 / 200$ & $\mathrm{PVC} / \mathrm{CI}$ & 456 & 6 & 6.8 \\
\hline
\end{tabular}

\subsection{Hydraulic model}

The pipe hydraulic model (1073 pipes and 986 nodes) was built up using the EPANET 2.0 system (Rossman, 2000) and the hydraulic head loss was calculated with the DarcyWeisbach equation and top-down demand approach. The time step for the hydraulic head loss calculation was set to $1 \mathrm{~s}$. The node demands were collected from a 6 month data survey for every connection. The daily patterns of consumption for inhabitants was set to $630 \mathrm{~m}^{3} \mathrm{day}^{-1}$. The daily pattern for companies and municipal organizations were set according to the information from the municipality of registered drinking water consumptions. There were two organizations in municipality, working constantly day and night, but the total amount of water consumption is only about $0.5 \mathrm{~m}^{3} \mathrm{~h}^{-1}$, thus, they did not influence the data generated by the model. The verification of water residence time was conducted by tracer studies (Fig. 3) since each well in the water treatment plant had a different electric conductivity (EC) in the range of $500-900 \mu \mathrm{S} \mathrm{cm}^{-1}\left(25^{\circ} \mathrm{C}\right)$.

All field measurements were recorded for several hours and were made from hydrants or at the taps in the buildings belonging to the drinking water supplier. For the field measurements, online instruments $3400 \mathrm{sc}^{\mathrm{TM}}$ with controller sc $100^{\mathrm{TM}}$ (Dr. Bruno Lange GmbH\&Co, Düsselfdorf, Germany) and condu::lyser ${ }^{\mathrm{TM}}$ with controller con::stat ${ }^{\mathrm{TM}}$ (s::can Meßtechnik GmbH, Vienna, Austria) supplemented with portable manual EC meter Cond 315i, (WTW, Germany) were used. The results of model verification are not shown in this study.

\subsection{Analysis of turbidity curves}

In order to generalize the trends of particle erosion observed during field experiments/UDF the following assumptions were made:

1. The hydraulic circumstances of the particle disturbance were based on the assumption that the accumulated particles erode and are transported by a plug flow. The distance $(L, \mathrm{~m})$ from hydrant outlet to location where erosion of particles starts was calculated with the formula below:

$$
L=\frac{4 \cdot Q \cdot t}{\pi \cdot D^{2}}
$$




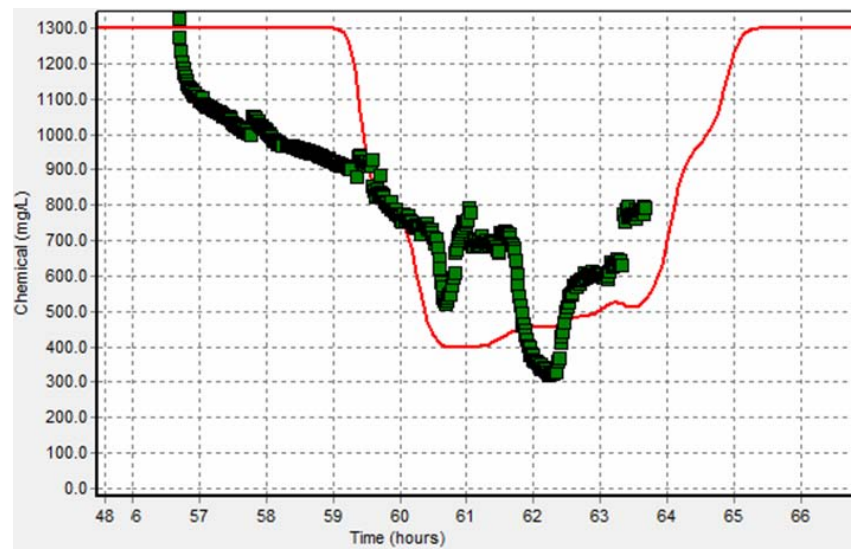

Figure 3. Measured (green squares) and modeled (red line) concentrations of the tracer near RPM measurement location No. 3 (see Fig. 1).

where $t$ is time (s), $Q-$ flushing flow $\left[\mathrm{m}^{3} \mathrm{~s}^{-1}\right]$ and $D$ - diameter of pipe $[\mathrm{m}]$. The same assumption has been used previously and in addition a term "locally settled sediment" (LSS) has been introduced (Blokker et al., 2010).

2. By using the principle of back-tracing, the location in networks corresponding to the spikes of $30 \mathrm{NTU}$ and 26 NTU in Fig. 4 were found using Eq. (1) and an estimates of flow.

3. The maximum value ( $30 \mathrm{NTU}$ and $26 \mathrm{NTU})$ of each spike was divided by the turbidity value just before spike, which means the average turbidity in straight section is (17 NTU of turbidity from beginning) $\operatorname{Turb}_{\max } / \operatorname{Turb}_{\mathrm{f}}=J$ coefficient. This value was used for the calculation of next peak magnitude, in case the upflow had some other fittings. Hence, a comparison of the difference between the particles originating from a linear pipe section and the particles which accumulated in the fittings could be compared. If a section is without fitting, spike, which comes with clear front (turnover), is not considered. As many data as possible are needed to create a database for several situations, according theoretical mass-balance from a treatment station.

A large number of data from flushing studies is needed to calculate a fitting coefficient for various conditions in order to predict the fouling of pipes with particles at the locations of T-pieces and valves.

\subsection{Modeling of particle erosion in drinking water network including fittings}

The stability of deposit layers is, according to the PODDS model, based on the hydraulically induced shear of the bulk water on the deposit surfaces. The daily maximum occurring

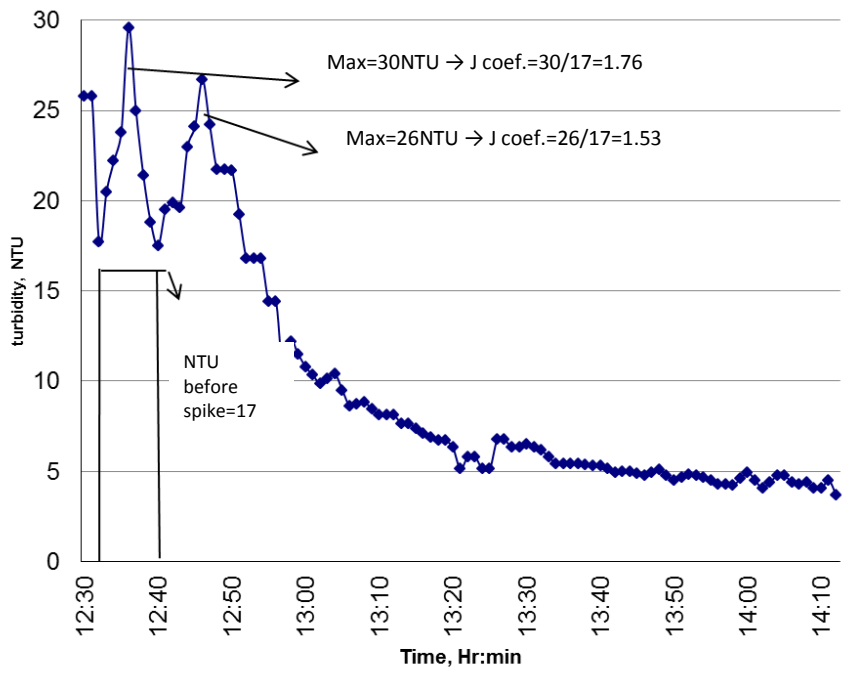

Figure 4. The turbidity pattern from UDF and schematic (graphical) representation for obtaining the algorithm for the $J$ coefficient for a certain location.

hydraulic wall shear stress is equivalent to the minimal shear stability of deposits according to a validated hydraulic model in the conditions of the maximum consumption of water per day; the maximal shear stress to pipe walls is calculated as follows:

$\tau=\rho g \frac{D}{4} \cdot l$

where $\tau$ is boundary shear stress $\left(\mathrm{N} \mathrm{m}^{-2} \mathrm{~m}^{-1}\right), \rho$ - density of water $\left(\mathrm{kg} \mathrm{m}^{-3}\right), g$ - gravity acceleration $\left(\mathrm{m} \mathrm{s}^{-2}\right), D$ - diameter of pipe $(\mathrm{m}), l=$ dimensionless energy gradient.

The turbidity curve predictions were calculated in a created algorithm (MS Excel). Using the PODDS model and substituting the equations therein, the output turbidity is equal to

$\Delta C_{\mathrm{c}}(t=1)=P_{\left(t_{\mathrm{a}}-t_{\mathrm{s}}\right)}^{n} \cdot 2 \pi r L$

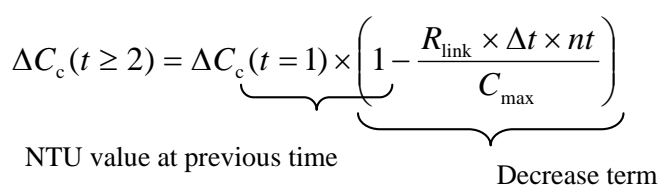

where $\Delta C_{\mathrm{c}}$ is the turbidity value at the current time at the flushing outlet. At time $t=1$ turbidity will have the maximum possible peak value for corresponding layer strength followed by gradual decrease of maximal peak value at $t \geq 2$ and so on. Character $\Delta t$ is the value of the time step in seconds, $P$ - gradient term $\left(\mathrm{NTU} \mathrm{m}^{-2}\right), n-$ power term $(-), t_{\mathrm{a}}$ - applied shear stress (NTU m $\left.{ }^{-2}\right), t_{\mathrm{s}}$ - current layer strength (NTU m $\left.{ }^{-2}\right), r$ - radius of a pipe (m), $L$ - length of a ling (m), $n t$ is number of current time step, $R_{\text {link }}$ is output peak turbidity for the corresponding link, NTU $\mathrm{Utat}_{\text {tol }}$ is the total accumulated mass following a flushing event. 
To predict the erosion of particles and obtain the character of the turbidity curve, the following equation was used:

$\Delta N=R \times A_{\mathrm{s}}$

where $\Delta N$ is the increase or decrease of turbidity (NTU), $R$ is the rate of supply (NTU m${ }^{-2}$ ), $A_{\mathrm{s}}$ is the pipe surface area $\left(\mathrm{m}^{2}\right)$.

Turbidity peak for plug flow will occur at some (current) time $C$, after which it will gradually decrease, described by NTU decrease term. The maximum peak value was calculated from the difference of the standard maximum velocities (hourly and flushing), in terms of corresponding shear stresses regardless of the mass accumulated in pipe. The total accumulated mass was used to calculate the total turbidity mass via linear daily shear stress-turbidity relationship, $\mathrm{NTU} \mathrm{m}^{3}=-475000 \tau_{\text {daily }}+529000$. Because of the total turbidity dimensions, the obtained value should be multiplied by the number of cubic meters in the pipe to obtain the total link turbidity and its maximal peak value. The magnitude rate of decrease depends on the coefficient: $P$ and exponent $n$ (variables in our case $P=0.1$ and $n=0.5$ ) in the rate of supply from the layer calculations:

$R=P\left(\tau_{\text {flush }}-\tau_{\text {daily }}\right)^{n}$.

Weak layers (according to shear stress stability) can store more turbidity units than strong layers. This means that pipes with a low flow rate have a higher potential of turbidity accumulation and the same factor is referable to the fittings. By generalizing the PODDS model, it can be said that in the conditions of a constant flushing flow and a constant shear stress, the output turbidity will have only one peak without considering the local turbulence in the fittings. As it was observed from the case study, after the first turbidity peak a second or third peak may follow, which often are higher than the first one. It was calculated that time of all the next peaks mainly conform to the time it takes the plug flow to travel from the fitting of a downstream pipe. Thus, in this study we have applied an additional term to the aforementioned turbidity equation in order to account for all peaks representing the downstream fittings.

$$
\begin{aligned}
& \text { Turbidity }(t \geq 2)=\mathrm{NTU}(t=1) \times\left(1-\frac{R \times t}{\mathrm{NTU}_{\text {total }}}\right) \\
& +\underbrace{\mathrm{NTU}_{\mathrm{f}} \times J \times\left(1-\frac{R \times t}{\left(Q \times t \times \mathrm{NTU} \times \mathrm{TSS} \times 10^{-6}\right)}\right)}
\end{aligned}
$$

Additional term describing the impact of a junction

where

$$
\begin{aligned}
& \mathrm{NTU}(t=1)=R \times A_{\mathrm{s}} \\
& \mathrm{NTU}_{\text {total }}=C_{\text {max }} /\left(\pi r^{2} L\right) \\
& \mathrm{NTU}_{\mathrm{f}}=\mathrm{NTU}_{\text {total }} / t
\end{aligned}
$$

$t$ is time (in seconds), $R$ - rate of supply (NTU m ${ }^{-2}$ ), $A_{\mathrm{s}}-$ pipe surface area $\left(\mathrm{m}^{2}\right), r$-radius of pipe $(\mathrm{m}), J$ - coefficient of a fitting, $L$ - length of pipe, $\mathrm{NTU}_{\mathrm{f}}$ denotes average output turbidity in first peak, $C$ - the stored turbidity, calculated from layer shear strength $\tau^{\prime}\left(\mathrm{NTU} \mathrm{m}^{3}\right)$.

\subsection{Calculations of particle mass}

The mass $(\mathrm{kg})$ of particles in a fitting was calculated using a following equation:

$M=\Sigma\left(Q \times t \times \operatorname{Turb} \times \mathrm{TSS} / 10^{6}\right)$

where $t$ is time (in seconds), $Q-$ flow rate $\left(1 \mathrm{~s}^{-1}\right)$, Turb the turbidity data from the online photometer (NTU), TSS the total suspended solids which was assumed as a constant (77.3 $\mathrm{mg} \mathrm{l}^{-1}$ ) for all the calculations (Boxall et al., 2003) where a wide range of samples obtained from different drinking water networks were analyzed.

In the Eq. (7) all the turbidity online measurements were summed up and each of the turbidity readings was converted to mass, using the above mentioned TSS constant, allowing the total mass of the flushing event to be calculated. It was assumed that the flushing plug flow velocity was equal to the flow velocity. Thus, by logging online discharge data, time from the start of the flushing event and knowing the diameters of the pipes located downstream from the flow velocity and thus past distances downstream from the pipe being flushed were calculated. As reported earlier, close similarities between online turbidity measurements peaks and downstream fitting were detected (Rubulis and Neilands, 2010).

\section{Results and discussion}

\subsection{Field experiments}

More than 67 isolated sections were flushed using UDF to collect turbidity data from washed-out particles. Both sections of CI, PVC and PE pipes were examined. The pipe diameters ranged from 100 to $300 \mathrm{~mm}$. The discharge from the hydrant was maintained constant to ensure constant shear stress on particles; therefore, the possible fluctuations in hydraulic conditions could take place only due to the changes in water production/consumption ratio. In Table 3 all data corresponding only to flushed sections with T-piece are summarized, since this type of fitting has most often been represented, instead of the bends of $30^{\circ}, 60^{\circ}, 90^{\circ}$, but those data were too few to make some valid approximation trends.

The total mass of washed out particles from T-pieces in this study ranged from 0.05 to $0.29 \mathrm{~kg}$. This mass was calculated by taking into account the relationship between turbidity and total suspended solids from studies of Boxall et al. (2003). The quantities of particles in our study were higher compared to the data from literature. The mass of about $0.0001-0.04 \mathrm{~g} \mathrm{~m}^{-1}$ was found to be present in four different Canadian networks (Carriere et al., 2005). The quantity of particles described by the other authors was even smaller: $0.00008 \mathrm{~g} \mathrm{~m}^{-1}$ (Jonas and Murphy, 2002) and 
Table 3. Description of T-pieces.

\begin{tabular}{|c|c|c|c|c|c|c|c|c|c|c|}
\hline Case study & $\begin{array}{l}V_{\text {day max }} \\
\left(\mathrm{m} \mathrm{s}^{-1}\right)\end{array}$ & $\begin{array}{l}\tau_{\text {day max }} \\
\left(\mathrm{N} \mathrm{m}^{-2}\right)\end{array}$ & $\begin{array}{l}V_{\text {flush }} \\
\left(\mathrm{m} \mathrm{s}^{-1}\right)\end{array}$ & $\begin{array}{l}\tau_{\text {flush }} \\
\left(\mathrm{N} \mathrm{m}^{-2}\right)\end{array}$ & $\begin{array}{l}\text { Junction } \\
\text { type }(\mathrm{mm})\end{array}$ & $\begin{array}{l}J \\
(-)\end{array}$ & $\begin{array}{l}\text { Mass in } \\
\text { junct. }(\mathrm{kg})\end{array}$ & $\begin{array}{l}V / D \\
(-)\end{array}$ & $\begin{array}{c}\text { Pipe } \\
\text { material }\end{array}$ & $\begin{array}{l}\text { Pipe length } \\
\text { (m) }\end{array}$ \\
\hline 1 & 0.21 & 0.15 & 0.43 & 0.59 & T-piece DN 200/100 & 1.87 & 0.24 & 1.05 & Cast iron & 457 \\
\hline 3 & 0.02 & 0.002 & 1.52 & 6.4 & T-piece DN 200/100 & 8.02 & 0.05 & 0.1 & Cast iron & 240 \\
\hline 4 & 0.39 & 0.22 & 0.37 & 0.45 & T-piece DN 250/250 & 2.23 & 0.2 & 1.56 & Cast iron & 300 \\
\hline 5 & 0.02 & 0.002 & 2.14 & 11.96 & T-piece DN 200/100 & 3.4 & 0.11 & 0.1 & PE & 159 \\
\hline 8 & 0.02 & 0.002 & 0.77 & 1.72 & T-piece DN 200/100 & 3.94 & 0.18 & 0.1 & Cast iron & 295 \\
\hline 9 & 0.27 & 0.24 & 1.13 & 3.6 & T-piece DN 250/150 & 5.6 & 0.17 & 1.08 & Cast iron & 204 \\
\hline 10.1 & 0.21 & 0.15 & 0.46 & 0.62 & T-piece DN 250/200 & 1.4 & 0.29 & 0.84 & Cast iron & 745 \\
\hline 10.2 & 0.21 & 0.15 & 0.46 & 0.62 & T-piece DN 150/150 & 2.75 & 0.22 & 1.4 & Cast iron & 400 \\
\hline 11.1 & 0.21 & 0.15 & 0.52 & 0.81 & T-piece DN 250/200 & 1.53 & 0.2 & 0.84 & Cast iron & 745 \\
\hline
\end{tabular}

$0.026 \mathrm{~g} \mathrm{~m}^{-1}$ (Delanoue and McMath, 1997). The conclusion in this study was that neither flushing $\left(0.37-2.14 \mathrm{~m} \mathrm{~s}^{-1}\right)$ nor daily maximum velocity $\left(0.02-0.39 \mathrm{~m} \mathrm{~s}^{-1}\right)$ correlated with the washout mass of the particles. It should be noted that a similar conclusion about flushing velocity $\left(0.65-2.3 \mathrm{~m} \mathrm{~s}^{-1}\right)$ and washed out mass of particles has previously been observed (Carriere et al., 2005). Analyses of the impact of daily maximum velocity on washed out mass of particles normally should be done by applying a careful hydraulic analysis using a "bottom-up" demand allocation principle, e.g. SIDMEUM (Blokker et al., 2008), rather than the "top-down" approach used in this study.

In the previous studies, the concentration of particles (or water turbidity) in distribution networks were studied depending on (1) source water quality and treatment process. Unfiltered systems have the greatest mass of solids compared to those applying filtration, with the mass decreasing based on source water and level of treatment (Ryan et al., 2008; Vreeburg et al., 2008); (2) the distance in the network, namely the increase of the particle concentration towards the mains dead ends (Gauthier et al., 2001, Matsui et al., 2007); the amount of deposits does not compulsorily increase at the end of pipe mains. If there are no suspended particles transported to the end of a pipe, which may settle, then there is also deposit formation; (3) the topography which is indirectly confirmed by the number of customer complaints about water quality in undulating terrains compared to those in flat terrains (Polychronopolous et al., 2003); (4) diameter of pipes where increased particle concentration in trunk mains, which in the network acts as sedimentation tanks, has been previously shown (Husband et al., 2010; Saldarriaga et al., 2010). (5) An incontrovertible factor that affects the concentration of particles is the maximum daily velocity, which can be re- lated to particles by applying "bottom-up" demand modeling, has been described earlier (Blokker et al., 2008). The same opinion is postulated in the cohesive transport modeling approach called PODDS (Boxall et al., 2001; Boxall and Saul, 2005; Husband and Boxall, 2010) which predicts that the particle deposits on pipe walls are conditioned by shear stress.

This conclusion is not fully consistent with the approach postulated in the PODDS since it may indicate that the particles will not accumulate in an annular manner. In this study, the asymmetrical accumulation of particles within the fittings was found, which is consistent with the scientific studies performed in other areas of research (Fokker et al., 2003). The flow structure and, specifically, vortex pairs formed downstream of the $90^{\circ}$ bend have been investigated in both micro-channel systems, e.g. $0.209 \mathrm{~mm}$ (Xiong and Chung, 2008), and pipe systems with diameters typical for drinking water mains, e.g. $150 \mathrm{~mm}$ (Peters and Ruppel, 2004). It should be noted that in both cases, the velocity was significantly higher than that found in drinking water networks, respectively $0.55-8.23 \mathrm{~m} \mathrm{~s}^{-1}$ (Xiong and Chung, 2008) and 8.3-31.1 $\mathrm{m} \mathrm{s}^{-1}$ (Peters and Ruppel, 2004), and in both cases a fully developed vortex formed while circular motion (outer/inner wall vortex after fittings) started to develop already at lower velocities which correspond to the conditions in drinking water systems. Some tracer studies have reported an incomplete mixing at the pipe fittings (e.g. cross and double T-piece) in water flow, and simulations with computational fluid dynamic (CFD) models at velocities $\left(0.35-1.4 \mathrm{~m} \mathrm{~s}^{-1}\right)$ have shown flow trajectories and possible vortex zones suitable for accumulation of particles after fittings (Ho et al., 2006; Webb and van Bloemen Waanders, 2006). Guided by the concept of particle rotational 


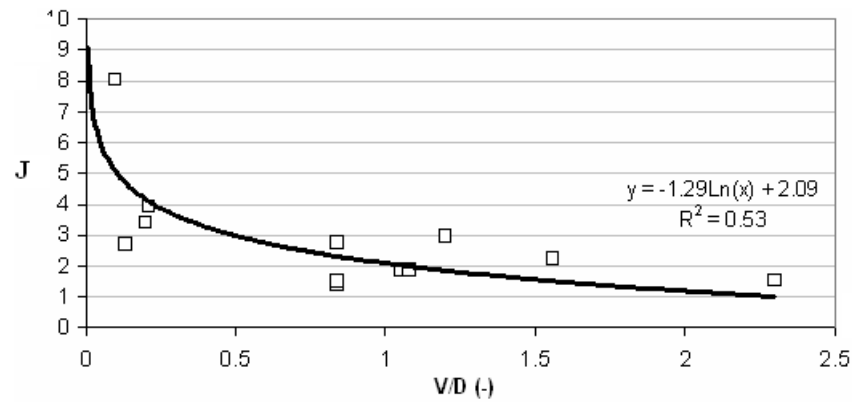

Figure 5. Turbidity potential of accumulated particles $(J)$ in Tpiece versus hydraulic conditions.

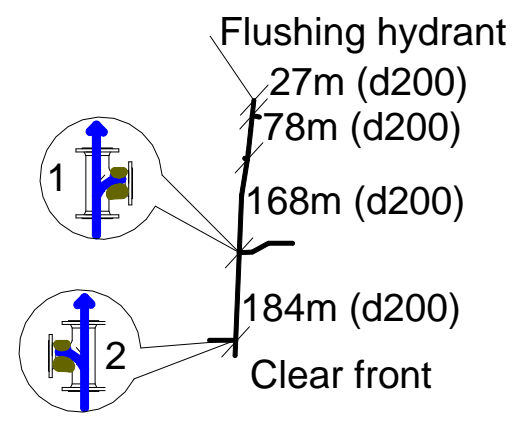

Figure 6. The section of CI pipe layout for site 1 (see Fig. 1). The locations of hydrant and fittings are indicated. The T-piece with reduction on right and left, respectively, to $100 \mathrm{~mm}$ is indicated under No. 1 and 2. The diameters of pipe segment in $\mathrm{mm}$ is shown in brackets.

trajectories in drinking water networks generated by inertia: the Magnus and the Saffman forces (van Thienen et al., $2011 \mathrm{~b}$ ), the development of radial processes in fittings instead of gravitational settling can be expected.

To generalize the observations from field experiments and to compare results of the erosion of accumulated particles in different T-pieces, the relationship between the maximum and average turbidity value from each specific section was calculated ( $J$ coefficient), which characterized the potential of the accumulated particles. The hydraulic conditions in specific T-piece were expressed in the relationship $V / D$ (maximal velocity per hour in typical day and diameter of inlet section of T-piece) as non-dimensional parameter.

With the increase of the maximum velocity per hour in the same fitting, the accumulated concentration of particles will decrease (Fig. 5). This relationship obtained from field experiments will supplement the knowledge presented in the PODDS model (Boxall et al., 2001).

$V / D$ reaches "self-cleaning" proportion, which is approximately $0.35 \mathrm{~m} \mathrm{~s}^{-1}$ (Blokker et al., 2010); theoretically, after that $J$ continues in a linear line with a value of 1 . The same function can be obtained for other types of fittings.

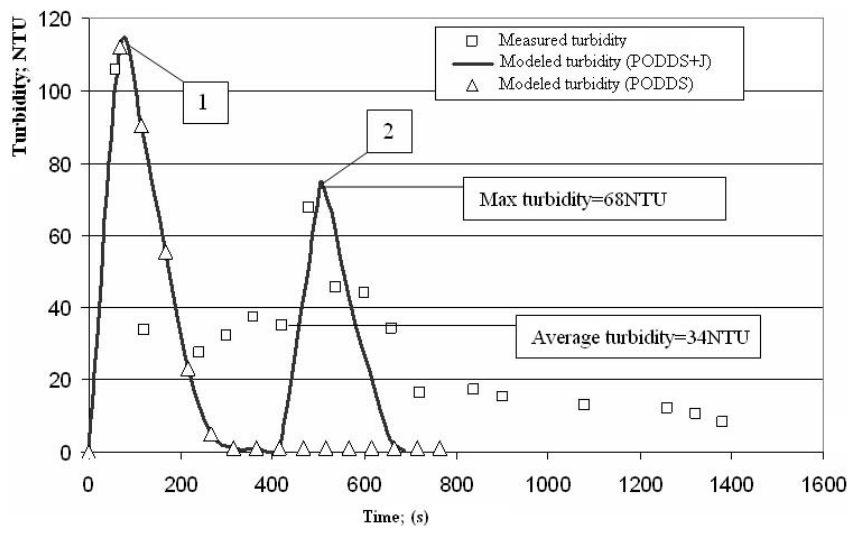

Figure 7. Turbidity curve for flushing site 1.

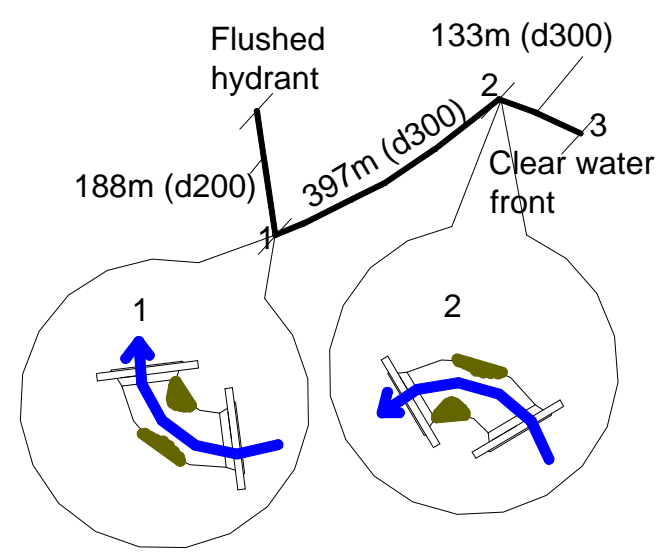

Figure 8. The section of CI pipe layout with two $90^{\circ}$ bends. The diameters in $\mathrm{mm}$ are shown in brackets.

\subsection{Modeling of particle erosion}

In order to model particle erosion from field experiments where network sections with T-pieces were flushed, an algorithm was added to the PODDS and the modeling results were compared with the original PODDS parameters. This improved modeling approach allows us to divide the modeled straight pipe sections in segments between the fittings and application of the coefficient for the potential particle accumulation $(J)$ in every step. The first site presented here had a typical straight section of CI transport main with a nominal diameter of $200 \mathrm{~mm}$ and two T-pieces for connections (Fig. 6). The location of this site is shown in Fig. 1 and other parameters which describe this site are shown in Table 3 as Case Study No. 1.

Figure 7 shows the temporal turbidity response within flushing, reaching two peaks: 110 and 68 NTU. Measured particle erosion was compared with the simulations of PODDS, which replicates only the rise and exponential decay of first peak of turbidity within the first 200 s of flushing. Introduction of the coefficient $J$ into PODDS and dividing 


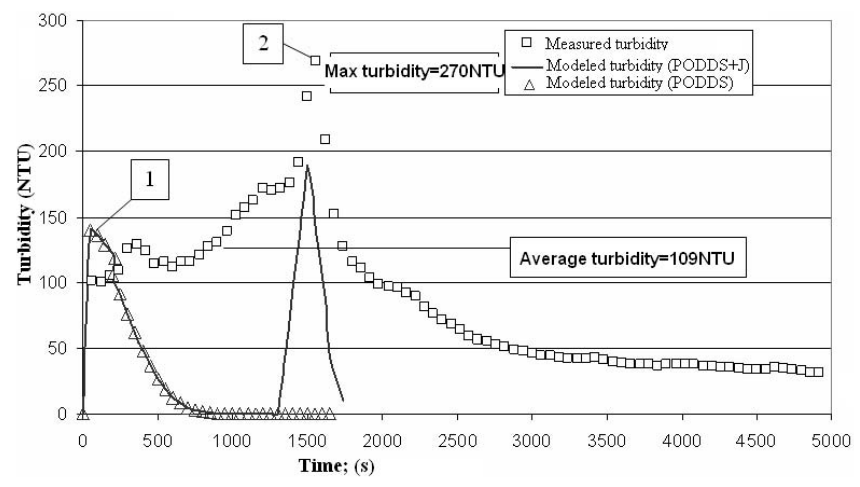

Figure 9. Turbidity curve for flushing site 2 containing two $90^{\circ}$ bends.

the simulated section in two segments allowed us to gain two peaks which approximately repeat measured data.

The second flushing site was chosen since it had two $90^{\circ}$ bends which are infrequently found in drinking water systems, unlike T-pieces (Fig. 8). The flushed section was $718 \mathrm{~m}$ long and consisted of sections of 200 and $300 \mathrm{~mm}$. The velocity obtained during flushing reached 0.57 and $0.87 \mathrm{~m} \mathrm{~s}^{-1}$, respectively.

The online turbidity results are shown in Fig. 9 which shows two visible turbidity peaks. The simulation results with PODDS and with PODDS supplemented with the coefficient $J$ are also shown here. Both simulation results only partially follow the magnitude of the first peak and display a much steeper decrease in turbidity compared to the measured values. The upgraded model, having coefficient $J$, is in a better agreement with online data compared to the original PODDS. The repeatability of accumulation of particles in fittings was proven with UDF in one CI pipe section of $1358 \mathrm{~m}$ length with $200 / 300 \mathrm{~mm}$ diameter two times within a 5 -month period, showing same pattern of turbidity (Rubulis and Neilands, 2010).

Due to the non-disclosure agreement, the authors of the PODDS do not present the values of model parameters $(k$, $b, P$ and $n$ ). Thus, in this study these were estimated using a trial and error approach. Some authors have attempted to replicate the erosion of particles using the PODDS approach and have used the following parameter values $C_{\max }=10$, $k=-0.15, b=1, P=0.0000065$ and $n=2.5$ (Aisopou et al., 2010) and $C_{\max }=5.0, k=-1.5, b=-2.5, P=0.0065$, $n=-3.5$ (Naser, 2006). By using the parameter values from Aisopou et al. (2010) and Naser (2006) in our case studies, the modeled results differed significantly. These results indicate that the parameters are site specific and therefore cannot be generalized.

Various aspects need to be improved in prediction and measurement of particle accumulation/erosion: the optical tomography presented by van Thienen et al. (2011b) can be one of the instruments; RPM could be updated based on the fitting quantity in a particular pipe section. Future work in this field should concentrate upon gaining a greater understanding of the development and formation of vortex or centrifugal forces in pipe fittings of drinking water distribution networks.

\section{Conclusions}

Measurements of turbidity, flow during UDF and analysis of pipe geometry showed significance of accumulation of particles in pipe fittings. Turbidity potential of accumulated particles $(J)$ was determined for T-pieces of several sizes from DN 100/100-DN 250/250 for CI and PE pipes. The existing turbidity model PODDS was updated with an additional term for pipe fittings $-(J)$. The total mass of particles accumulated in T-pieces ranged from 0.05 to $0.29 \mathrm{~kg}$. The study showed that pipe fittings (T-pieces, valves, bends etc.) act as catchment areas for particle accumulation in drinking water networks.

\section{Appendix A}

\section{Notation}

\begin{tabular}{|c|c|}
\hline$A_{\mathrm{s}}$ & pipe surface area $\left(\mathrm{m}^{2}\right)$ \\
\hline C & $\begin{array}{l}\text { stored turbidity, calculated from layer } \\
\text { shear strength }\end{array}$ \\
\hline $\begin{array}{l}\Delta C_{\mathrm{c}} \\
D\end{array}$ & $\begin{array}{l}\text { changes in turbidity potential }\left(\mathrm{NTU} \mathrm{m}^{2} \mathrm{~s}^{-1}\right) \\
\text { diameter of pipe }(\mathrm{m})\end{array}$ \\
\hline$f$ & $\begin{array}{l}\text { a dimensionless coefficient called } \\
\text { the Darcy friction factor }\end{array}$ \\
\hline$g$ & gravity acceleration $\left(\mathrm{m} \mathrm{s}^{-2}\right)$ \\
\hline$\Delta H$ & the head loss due to friction $\left(\mathrm{mH}_{2} \mathrm{O}\right)$ \\
\hline$J$ & $\begin{array}{l}\text { coefficient of a fitting/turbidity potential } \\
\text { of accumulated particles }\end{array}$ \\
\hline$L$ & the length of the pipe $(\mathrm{m})$ \\
\hline$M$ & mass of particles $(\mathrm{kg})$ \\
\hline$\Delta N$ & in/decrease of turbidity (NTU) \\
\hline$Q$ & flow rate $\left(\mathrm{m}^{3} \mathrm{~s}^{-1}\right.$ or $\left.1 \mathrm{~s}^{-1}\right)$ \\
\hline$r$ & radius of pipe $(\mathrm{m})$ \\
\hline$R$ & rate of supply $\left(\mathrm{NTU} \mathrm{m}{ }^{-2}\right)$ \\
\hline$t$ & time $(s)$ \\
\hline TSS & total suspended solids $\left(\mathrm{mg} \mathrm{l}^{-1}\right)$ \\
\hline$V$ & the average velocity of the fluid flow $\left(\mathrm{m} \mathrm{s}^{-1}\right)$ \\
\hline$\tau$ & boundary shear stress $\left(\mathrm{N} \mathrm{m}^{-2} \mathrm{~m}^{-1}\right)$ \\
\hline$\rho$ & density of water $\left(\mathrm{kg} \mathrm{m}^{-3}\right)$ \\
\hline$\pi$ & $\begin{array}{l}\text { a mathematical constant } \\
\text { approx. equal to } 3.14\end{array}$ \\
\hline
\end{tabular}

Acknowledgements. This work has been supported by the European Social Fund within the project "Support for the implementation of doctoral studies at Riga Technical University".

This work has been undertaken as a part of the research project "Technology enabled universal access to safe water - TECHNEAU" 
(Nr. 018320) which is supported by the European Union within the 6th Framework Programme.

There hereby follows a disclaimer stating that the authors are solely responsible for the work. It does not represent the opinion of the Community and the Community is not responsible for any use that might be made of data appearing herein.

The authors would like to acknowledge the help of Talis Juhna for his guidance throughout the method development for the measurement of turbidity in field. The authors are thankful to Sandis Dejus for assistance in calibration of the hydraulic model and the director of Adazi udens Ltd. Janis Neilands for his advice and guidance during the field campaign. Special thanks must be expressed to Edgars Grundbergs and Janis Neilands Jr. for their assistance in the flushing and tracer campaigns. Special thanks are due to Simona Larsson for reviewing the English of this paper.

Edited by: T. Juhna

\section{References}

Aisopou, A., Stoianov, I., and Graham, N.: Modelling Discoloration in WDS Caused by Hydraulic Transient Events, in: Proceedings of the Water Distribution System Analysis Conference, Tucson, Arizona, ASCE Conf. Proc., doi:10.1061/41203(425)49, 2010.

Blokker, E., Vreeburg, J., Schaap, P., and van Dijk, J.: The selfcleaning velocity in practice, in: Proceedings of the Water Distribution System Analysis Conference, Tucson, Arizona, ASCE Conf. Proc., doi:10.1061/41203(425)19, 2010.

Blokker, E. J. M., Vreeburg, J. H. G., Buchberger, S. G., and van Dijk, J. C.: Importance of demand modelling in network water quality models: a review, Drink. Water Eng. Sci., 1, 27-38, doi:10.5194/dwes-1-27-2008, 2008.

Boxall, J. B. and Saul, A. J.: Modeling discoloration in potable water distribution systems, J. Environ. Eng., 131, 716-725, 2005.

Boxall, J. B., Skipworth, P. J., and Saul, A. J.: A novel approach to describing sediment movement in distribution mains, based on measured particle characteristics, Water Softw. Syst., 1, 263273, 2001.

Boxall, J. B., Saul, A. J., Gunstead, J. D., and Dewis, N.: Regeneration of discolouration in distribution systems, World Water and Environmental Resources Congress, Philadelphia, ASCE Conf. Proc., 434-442, doi:10.1061/40685(2003)132, 2003.

Carriere, A., Gauthier, V., Desjardins, R., and Barbeau, B.: Evaluation of Loose Deposits in Distribution Systems through Unidirectional Flushing, J. AWWA, 97, 82-92, 2005.

Delanoue, A. and McMath, S. v. M.: Effect of Pipe Materials in Biofilm Growth and Deposition Formation in Water Distribution Systems, Proc. Of the Water Quality Technology Conference, Denver, Colo., AWWA, 1997.

Fokker, S., Kingman, S., Lowandes, I., and Reynolds, A.: Characterisation of the cross sectional particles concentration distribution in horizontal dilute flow conveying - a review, Chem. Eng. Process., 43, 677-691, 2003.

Gauthier, V., Gerard, B., Portal, J.-M., Block, J.-C., and Gatel, D.: Organic matter as loose deposits in a drinking water distribution system, Water Res., 33, 1014-1026, 1999.
Gauthier, V., Barbeau, B., Millette, R., Block, J.-C., and Prévost, M.: Suspended particles in the drinking water of two distribution systems, Wa. Sci. Technol., 1, 237-245, 2001.

Ho, C. K., Orear Jr., L., Wright, J. L., and McKenna, S. A.: Contaminant Mixing at Pipe Joints: Comparison between Laboratory Flow Experiments and Computational Fluid Dynamics Models, in: Proceedings of the Water Distribution System Analysis Symposium, Cincinnati, Ohio, ASCE Conf. Proc., doi:10.1061/40941(247)153, 2006.

Husband, S. and Boxall, J. B.: Field studies of discoloration in water distribution systems: model verification and practical implications, J. Environ. Eng., 36, 86-94, 2010.

Husband, S., Williams, R., and Boxall, J. B.: Discolouration risk management for trunk mains, in: Proceedings of the Water Distribution System Analysis Conference, Tucson, Arizona, ASCE Conf. Proc., doi:10.1061/41203(425)50, 2010.

Husband, P. S., Boxall, J. B., and Saul, A. J.: Laboratory studies investigating the processes leading to discolouration in water distribution networks, Water Res., 42, 4309-4318, 2008.

Jonas, S. and Murphy, B.: Distribution System Flushing Optimization to Reduce Environment Impact, Proc. Of the Water Quality Technology Conference, Seattle, AWWA, 2002.

Matsui, Y., Yamagishi, T., Terada, Y., Matsushita, T., and Inoue, T.: Suspended particles and their characteristics in water mains: development of sampling methods, J. Water Supply Res. T., 56, 13-24, 2007.

Naser, G.: Water quality transformations in pipelines: a twodimensional multi-component simulation model, Academic dissertation, University of Toronto, 2006.

Neilands, K., Rubulis, J., and Juhna, T.: Application of Online Monitoring, Resuspension Potential Method and Unidirectional Flushing for Control of Drinking Water Quality in a Distribution Networks, Latvia, in: Proceedings 1st Eastern European Regional Young Water Professionals Conference, Minsk, Belarus, ISBN 987-985-525-145-4, 2009.

Peters, F. and Ruppel, C.: A pressure probe for the detection of the flow direction close to walls, Case study: flow through a bend, Exp. Fluids, 36, 813-818, 2004.

Polychronopolous, M., Dudley, K., Ryan, G., and Hearn, J.: Investigation of factors contributing to dirty water events in reticulation systems and evaluation of flushing methods to remove deposited particles., Wa. Sci. Technol, 3, 295-306, 2003.

Rossman, L. A.: EPANET 2 Users Manual, EPA/600/R-00/057, US Environmental Protection Agency, Cincinnati, OH, 2000.

Rubulis, J. and Neilands, K.: Interpretation of loose deposits motion in drinking water distribution network, in: Proceedings of the Water Distribution System Analysis Conference, Tucson, Arizona, ASCE Conf. Proc., doi:10.1061/41203(425)61, 2010.

Ryan, G., Mathes, M., Haylock, G., Jayaratne, A., Wu, J., NouiMehidi, N., Grainger, C., and Nguyen, B. V.: Particles in Water Distribution Systems, Tech. Rep. 33, Cooperative Research Centre for Water Quality and Treatment, 2008.

Saldarriaga, J., Nieto, L., Hernández, F., and Osorio, C.: Discolored Water Analysis and Modeling for Trunk Networks: The Case of Bogotá, Colombia, in: Proceedings of the Water Distribution System Analysis Conference, Tucson, Arizona, ASCE Conf. Proc., doi:10.1061/41203(425)48, 2010.

van Thienen, P., Vreeburg, J. H., and Blokker, E. J.: Radial transport processes as a precursor to particle deposition in drinking water 
distribution systems, Water Res., 45, 1807-1817, 2011a.

van Thienen, P., Floris, R., and Meijering, S.: Application of optical tomography in the study of discolouration in drinking water distribution systems, Drink. Water Eng. Sci., 4, 61-69, doi:10.5194/dwes-4-61-2011, 2011b.

Vreeburg, J. H. G.: Discolouration in drinking water systems: a particular approach, Academic dissertation, Delft University of Technology, Delft, p. 183, 2007.

Vreeburg, J. H. G., Schaap, P., and van Dijk, J. C.: Measuring Discoloration Risk: Resuspention Potential Method, IWA Leading Edge Conference, Prague, 2004.

Vreeburg, J. H. G., Schippers, D., Verberk, J. Q. J. C., and van Dijk, J. C.: Impact of particles on sediment accumulation in a drinking water distribution system, Water Res., 42, 4233-4242, 2008.
Verberk, J. Q. J. C., Hamilton, L. A., O'Halloran, K. J., van der Horst, W., and Vreeburg, J. H. G.: Analysis of particle numbers, size and composition in drinking water transportation pipelines: results of online measurements, Wa. Sci. Technol., 6, 35-43, 2006.

Webb, S. W. and van Bloemen Waaders, B. G.: High fidelity computational fluid dynamics for mixing in water distribution systems, in: Proceedings of the Water Distribution System Analysis Symposium, Cincinnati, ASCE Conf. Proc., doi:10.1061/40941(247)154, 2006.

Xiong, R. and Chung, J.: Effects of miter bend on pressure drop and flow structure in micro-fluidic channels, Int. J. Heat Mass Tran., 51, 2914-2924, 2008. 